\title{
"Naked and on Fire": Examining Player Agency Experiences in Narrative-Focused Gameplay
}

\author{
Elin Carstensdottir \\ ecarsten@ucsc.edu \\ University of California, Santa Cruz \\ Santa Cruz, California \\ Ryan Williams \\ williams.ry@northeastern.edu \\ Northeastern University \\ Boston, Massachusetts
}

\author{
Erica Kleinman \\ emkleinm@ucsc.edu \\ University of California, Santa Cruz \\ Santa Cruz, California \\ Magy Seif El-Nasr \\ mseifeln@ucsc.edu \\ University of California, Santa Cruz \\ Santa Cruz, California
}

\begin{abstract}
Player agency is central to interactive narrative and games. While previous work focuses on analyzing player perception of agency through various lenses and phenomena, like meaningful choice and expectations, it is largely theoretical. Few user studies within games explore how players reason about and judge their own agency within interactive narratives. We present an interview study where participants rated their agency experiences within narrativefocused games and described their reasoning. The analysis suggests that agency perception depends on multiple factors beyond meaningful choice, such as social investment and genre-conventions. Participants described varying preferences and value judgements for different factors, indicating that individual differences have a deep impact on agency perception in narrative-focused gameplay. We discuss the implications of these cognitive variables on design, how they can be leveraged with other factors, and how our findings can help future work enhance and measure player agency, within interactive narrative and beyond.
\end{abstract}

\section{CCS CONCEPTS}

- Human-centered computing $\rightarrow$ Empirical studies in $\mathrm{HCI}$; Applied computing $\rightarrow$ Computer games.

\section{KEYWORDS}

player agency, user experience, interactive narrative, player experience, video games, storytelling

\section{ACM Reference Format:}

Elin Carstensdottir, Erica Kleinman, Ryan Williams, and Magy Seif ElNasr. 2021. "Naked and on Fire": Examining Player Agency Experiences in Narrative-Focused Gameplay. In CHI Conference on Human Factors in Computing Systems (CHI '21), May 8-13, 2021, Yokohama, Japan. ACM, New York, NY, USA, 13 pages. https://doi.org/10.1145/3411764.3445540

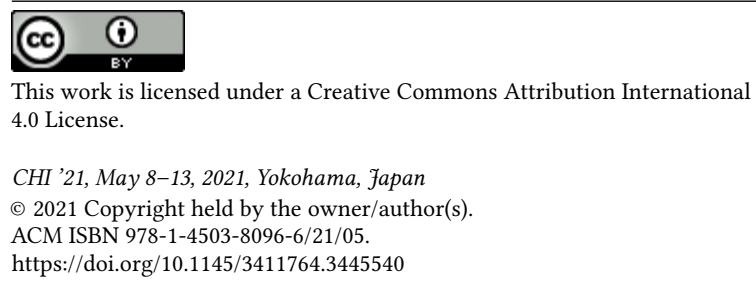

\section{INTRODUCTION}

Player agency is defined in a myriad of ways. Broadly, agency in games can be described as the phenomenon where a player feels that the actions presented to them in the context of the game are meaningful and that their choice of action has a meaningful impact on the context in which they are engaging. Previous work has been largely theoretical or inspired by design practice, focusing on defining and conceptualizing what agency is and how it manifests in relation to narrative interaction and gameplay e.g. $[1,8,18,24,26,27,32,38]$. Studies of player experience of agency in games have focused on the impact of choices and agency in narrative-focused games $[3,12,29]$ but have not examined narrative agency from the perspective of how players experience it more broadly. Lack of knowledge on how players themselves perceive and attribute agency within the context of the narrative experience makes it difficult to design applications with the intention of generating sensations of high agency. This is paramount for high impact domains such as educational games, as there is lack of clarity and understanding of how players perceptions of agency can confound designs meant to encourage student autonomy and might even confound comprehension of the material. Further still, if the intention is to create a low agency experience, for instance for experimental purposes, understanding of how players will perceive or reason about narrative agency is required.

To further our understanding of player agency, and assist designers in accounting for and designing their games for increased narrative agency, we need further study of how players themselves experience narrative agency. Specifically, we must focus on how they reason about and attribute it to specific design features, and whether there are broader constructs that can be leveraged to evaluate and measure agency more broadly.

Towards this end, we conducted an interview study in which participants $(n=28)$ were prompted to rate narrative driven games in terms of how much agency they felt, and then asked to justify or explain their rating. Specifically, we focused on agency as it was perceived or felt by the players, which we acknowledge as different from agency as afforded by the game's design. Through thematic analysis and iterative coding, we examine what factors players associate with their experience of agency in narrative driven video games, with the goal of gaining further understanding of how these factors contribute and shape the player's perception of agency. Our findings suggest that agency is a dynamic and nuanced 
phenomenon, and that perception of agency is highly susceptible to individual differences. Further, while some factors appeared to be more dominant contributions to perceived agency than others, no one factor appeared to exclusively dictate perception of the experience. To our knowledge, this is the first phenomenological study of player agency perception for interactive narrative in games. As such, this work broadens current knowledge of both design implications of player agency perception and offers insights into how to better study and measure player agency for future work.

\section{PREVIOUS WORK}

The interactivity afforded by digital media, specifically games and interactive narratives [5], disrupts traditional notions of storytelling by granting the player the ability to take actions that influence the progression of events $[7,17]$. The ability of the player to take actions that influence the story has broadly been referred to as giving the player agency within the story. More specifically, one of the first known definitions of player agency within game design literature defines agency as "the satisfying power to take meaningful action and see the results of our decisions and choices" [26]. Player agency is a central topic to both games and interactive narrative, and has been discussed extensively in the community $[1,2,4,8,14,18,23$, $24,26,30,32,34,37,38]$.

A number of conflicts arise as result of the relationship between interaction, agency, and coherent storytelling, and originates in the manner through which agency is defined or conceptualized within a game or storytelling framework. Such conflict has driven the discourse around player agency. One such conflict centers around the assumption that unrestricted player agency was highly desirable, which creates an inherent dilemma for narrative-driven gameplay where the author (or computational system) may be reluctant to relinquish control over narrative progression [17, 18, 22]. To address this tension between the player and the author of the story, designers and researchers developed various design methods and intelligent systems, such as drama managers [21, 28]. However, this indicates that player agency was seen as a phenomena to be managed and controlled for.

In response, researchers have proposed additional work to broaden how agency is defined in relation to play and narrative, the roles and relationship of the player and the author, and how it manifests in response to design.

Harrell and Zhu [18] argue that narrative agency is contextually situated, and that both the player and the system must be taken into account when agency is considered within interactive narratives. They further stipulate that agency is mediated through the player's interpretation of system behavior and affordances of the player interaction. They demonstrate how agency can be manipulated to convey narrative meaning in their work on Memory: Reverie Machine, where the player gradually loses control over a game character, and therefore agency to influence narrative progression, as the character gains its own self awareness [39].

Wardrip-Fruin et al. likewise argue that agency involves both the player and the game and, building on previous work [24], further define agency as occurring "when the actions players desire are among those they can take (and vice versa) as supported by an underlying computational model." [38]. They argue that understanding of and accounting for player expectations is required for successful play experiences, and in which the player's expectations are developed and/or adapted during gameplay as the player builds mental models of the internal workings of the system.

Complementary to this work, is Tanenbaum and Tanenbaum's conceptualization of agency as commitment to meaning [31, 32]. They propose that "agency is better understood as a commitment to meaning instead of a desire to act freely.", and as such is more about communication through meaningful mappings between player and the game system $[31,32]$ where the player is able to express intent and have the game provide a satisfying response to that intent.

These are only 3 of several examples of how player agency has been defined and conceptualized for interactive narrative and games. Like these examples, most work on player agency in games has remained largely theoretical. Current related work has not claimed to enumerate the multiple dimensions through which players might experience agency during gameplay or in relation to interactive narrative. Researchers have explored how various elements of game experiences impacts agency [7,11], and how agency can be manipulated to convey meaning through gameplay [25, 39]. Additionally, while insightful and in some cases based in design practice, these definitions and theories have yet to be formally validated.

Several studies have aimed at improving formal understanding of how agency is perceived or experienced by players in games and interactive narratives. Studies have examined the relationship between agency and flow [36], and explored agency in relation to interaction with storytelling systems at the level of its emotional and aesthetic context [16]. Fendt et al. [12] evaluated whether acknowledgement of player choices that did not influence gameplay had the potential to preserve the player's sense of agency in an interactive narrative. Cardona-Rivera et al. [4] found that players reported higher feelings of agency when faced with choices perceived to result in meaningfully different states, in contrast to choices perceived to result in similar states. Roberts et al. [29] found that influencing a player's decision making was better at guiding players towards the author's intended goal, but that players felt more in control of the narrative without it. While these studies are comprehensive and their results are informative, they all focus on player choice, thus examining very specific aspects of the player experience. They do not explore other constructs relating to the player experience such as expectation or communicative meaning as proposed by Wardrip-Fruin et al. and Tanenbaum and Tanenbaum.

Beyond choice, Cole and Gillies's [10] study of avant-garde games identified narrative agency as a strong theme relating to overcoming emotional and other in-game challenges, but, as the authors state, it "did not fit into the commonly understood meaning of "agency"". They define two dimensions of player agency to describe their findings: Actual to Interpretive, and Fictional to Mechanical. However, their findings do not provide a sufficiently detailed and comprehensive account of which individual design elements and specific factors players associate with narrative agency, which is the primary goal of this work.

Most relevant to the work presented here is work on identifying contributing factors of agency. Revi et al's review of the interactive narrative literature identified 6 dimensions of agency: autonomy, 
effectance, control, manipulation, personalization, and usability [27]. Mallon's taxonomy of player agency is based on focus-group studies exploring perception of agency in narrative dimensions of adventure and role-play in computer-game design [22]. This work differs in several ways. In Mallon's study, participants were instructed to discuss a limited number of games, selected based on specific criteria, restricting the results to the chosen games and genres. In our study, participants were free to choose any games they had played recently that they thought were narrativefocused. Further, the study focused on single player games, whereas participants in our work were able to include multi-player games if they so chose. Finally, Mallon's study was a focus group, allowing participants to converse with one another, whereas our study was conducted individually. We argue that performing the study in an individual manner allows us to better identify individual differences.

Proposals have been made towards building a more theory driven approach to player agency for interactive storytelling technologies, specifically for experience managers [34]. Thue et al. [34] building on work by Thompson et al. [33] on control heuristics, propose a computational model for perceived player agency. An evaluation study showed that their computational model could predict with $96 \%$ confidence which sub-trees of a story afforded the player with higher perceived agency [35].

Ultimately, agency remains a highly contested topic which has been defined and conceptualized in a variety of ways, with some arguing that player agency is an illusion and exists as perceived interpretation of experience [30]. This highlights the ongoing discussion of the nature of agency as afforded by the game itself versus the perception and feeling of having it as a player.

Obtaining a comprehensive understanding of how players perceive agency, and what design elements impact that perception, remains an open problem due to a lack of phenomenological studies on the topic. In this study we examine what factors players associate with their experience of agency in narrative driven video games, with the goal of gaining further understanding of how these factors contribute and shape the player's perception of their agency. This study aims to capture how players themselves conceptualize and verbalize their experience of agency during gameplay by focusing on having players self-report their perceived level of agency in the game, and then have them justify as to why that level of agency was specified.

\section{METHODOLOGY}

To explore how players perceive, experience, and reason about agency, we conducted an interview study where players described their experiences playing narrative games. We then analyzed the resulting data to identify what design elements and other phenomenon typically impacted players' perceptions of agency during gameplay.

We took a constructivist approach, instead of providing a strict definition, we leaned more towards grounded theory/phenomenology where we sought to define the concept based on the data i.e. how players described it. This is different from the majority of previous work which has focused on defining agency from a theoretical point of view. As such, information was kept to a minimum so as to not bias the participants' existing conceptualization of agency.

\subsection{Participant Recruitment}

Participants were identified and recruited from the student populations at several Universities in the North Eastern United States, special interest groups around game design and play, and the researchers' online social networks. All participants were recruited with a predetermined recruitment script, delivered either in-person or online. Participants were required to be over 18 years of age, capable of communicating in spoken English, and have played at least a few story driven video games. No other inclusion or exclusion criteria were used. Participants were not compensated, and the study was a strictly voluntary activity. A total of 28 participants were recruited in the study.

\subsection{Interview Protocol}

Data was gathered via one-on-one semi-structured interviews with either one or both researchers present. After giving consent, and providing brief demographic data regarding video game experience and preferences, participants were asked whether they knew what agency was. If they did not, they were provided with the following definition: "Agency is the sense of control you feel you have over the game story and your interaction with the game as a whole." If participants claimed they knew what it was, the interviewer did not provide the definition unless explicitly asked. Each participant was then asked to name narrative driven games, or games that had narrative in them, that they had played recently. For each of these games, they were asked to rate the game as having low, medium, or high agency, and justify the rating by describing, in as much detail as possible, what game elements they believed impacted their agency. Their descriptions were transcribed verbatim by the attending researcher. Participants were asked to discuss at least three games and were allowed to change their overall agency rating for any of the games at any time during the interview process. Participants were then asked to describe what they believed constituted a high and a low agency experience. After completion of these steps, each participant was debriefed and thanked for their participation. Interviews lasted approximately one hour on average, and up to two hours at most.

\subsection{Data Processing}

Participant interview responses were segmented by line [6], determined by how the text lined up in a generic text editor, and organized in a separate document such that each response was presented along with the participant ID, name of the game, and agency rating. The text was segmented by line rather than by sentence as sentence by sentence coding proved too unwieldy for large, run-on sentences, which were common in the data set. All references to specific game titles and game genres were identified and labeled with either [game] or [genre] in the text. This was for clarification, in case a coder was not familiar with the games or genres in question. Additionally, coders were instructed to look up definitions for unfamiliar terminology.

\subsection{Data Analysis}

The interview data was analyzed via an iterative coding process consisting of an initial round of thematic analysis [15] to construct the first code book, followed by four rounds of coding. The first 
two rounds were conducted by two and four coders respectively, the last two rounds were conducted by two coders. For all rounds following the first round, coders were instructed to first familiarize themselves with the code book, which was updated for each round Coders then analyzed the data line by line, applying from zero to three codes per line, as they deemed appropriate. After each round, coders gave feedback on the code book such as clarification of phrasing or examples, which were then used to update the code book for the subsequent iteration. For the third and fourth iterations, inter-rater reliability was calculated via Cohen's Kappa [9] to assess the agreement in the coding and to identify inconsistencies or lack of clarity in the code book. For every line in the data, if the coders applied the same code, the agreement was counted as 1 for that code for both coders. If the coders did not apply the same code, but both applied the same number of codes, each disagreement was counted once. If no code was applied, or one coder applied less codes than the other, it was considered a disagreement between whatever code was applied and "no code". In the third round, the inter-rater reliability was calculated at .56 , too low to indicate satisfactory agreement. The researchers thus discussed their applications to identify confounding codes and unclear definitions, which were then merged or further clarified in the code book. In the fourth and final round, inter-rater reliability was .72, indicating strong agreement [20].

Participants descriptions of high and low agency experiences, which were collected as part of the interviews, were utilized during the development of the code book. They captured further information about what participants explicitly associated with high and low agency experiences respectively. However, the purpose of these descriptions was not to have participants provide definitions of agency but to articulate the elements they most strongly associated with changing or influencing their perception and feelings of agency. These descriptions were therefore helpful to highlight a prioritized set of elements that participants more strongly associated with either ranking. They were not found to include any new information or discussion that was not already present in the interview data upon the initial thematic analysis. Therefore they were used exclusively as references and additional material for the code book development. In future work we aim to explore the association between game elements and high and low agency perceptions in greater detail.

\section{RESULTS}

\subsection{Participants}

Data was collected from interviews with 28 participants, with an age range between 19 and 32 years. While the majority of participants were students, $30 \%$ (8 participants) were not. All participants were based in the US at the time of their interview. At least 6 participants mentioned during interviews to have grown up outside the US ( 2 in Europe, 3 in the Middle East, and 1 in Asia). Participants represented a variety of preferences in their game play experience, which covered all popular modes and genres of video game play. Participants used 2-3 different platforms on average to play on (the most common pairing was a personal computer and a console).

Participants played games with varying frequency, with the most common being spending more than six hours on playing games per week (17 participants). All but two participants explicitly mentioned narrative as being one of their main enjoyments during video game play. The number of games provided by each participant ranged from two to 28, with participants providing descriptions of five to six (5.43) games on average. If more than 10 games were provided, only the first 10 games described by the participant were included in the analysis. This was to minimize skew from participants that provided a significantly larger set of game descriptions. Due to the variety and number of games described, there was not a significant number of descriptions of any particular game.

Participants were all given an identification code consisting of the letter i and a number (i1, i2 etc.).

\subsection{Code Book}

The code book defined 17 codes that capture elements or phenomena that relate to how participants reported on perceiving and reasoning about their agency. These were: Structure, Choices, Endings, Narrative Impact, Mechanical Experience, Plot Twist, Preferences, Expectations, Genre Conventions, Customization, Emotional Investment, Social Investment, Story Quality, Meta-Acknowledgement, Player Narrations, Rating Statement, and Comparisons.

Of these codes, four referred directly to structural elements and the perceived impact associated with them (Structure, Choices, Endings, Narrative Impact), four referred to player experience factors (Emotional Investment, Social Investment, Preference, Rating Statement), three focused on factors pertaining to player knowledge (Genre Conventions, Expectations, Comparison), three referred to factors exclusive to the story content (plot twist, story quality, meta-acknowledgement), two referred to the player experience as it related specifically to mechanical experience and customization (mechanical experience, customization), and finally, one referred to factors pertaining to how players were able to narrate and make the experience their own (player narrations).

\subsection{Structure and Narrative Impact}

The "Structure and Narrative impact" category contains 4 factors: Structure, Choices, Endings, and Narrative Impact. See table 1 for code definitions. These factors all cohesively refer to the core interaction components of interactive narratives. They encapsulate the elements and concepts relating to the manner in which the story was perceived to be organized and impacted by the player's interaction. Additional concepts that are encompassed include the means and manner of interaction the players perceived themselves to have when interacting with the story.

Structure was applied whenever a participant mentioned the organization of presentation and interaction opportunities for the player i.e. perceived event ordering, sequencing, and other causal relationships used to present a game's story content and the interaction the player is afforded. Structure does not refer to content such as plot or character dialogue. Structure was applied most frequently of all the codes, a total of 228 times. Participants often used known structure patterns, like "linear" and "branching", in relation to structure. For example: "The story is pretty linear, as in there's a core arc that you're going over, but the part of it that I've enjoyed the most is each of the side quests, they have a fair amount of branching in them" (i10, The Witcher 3). Those participants that did 


\section{Play Frequency}

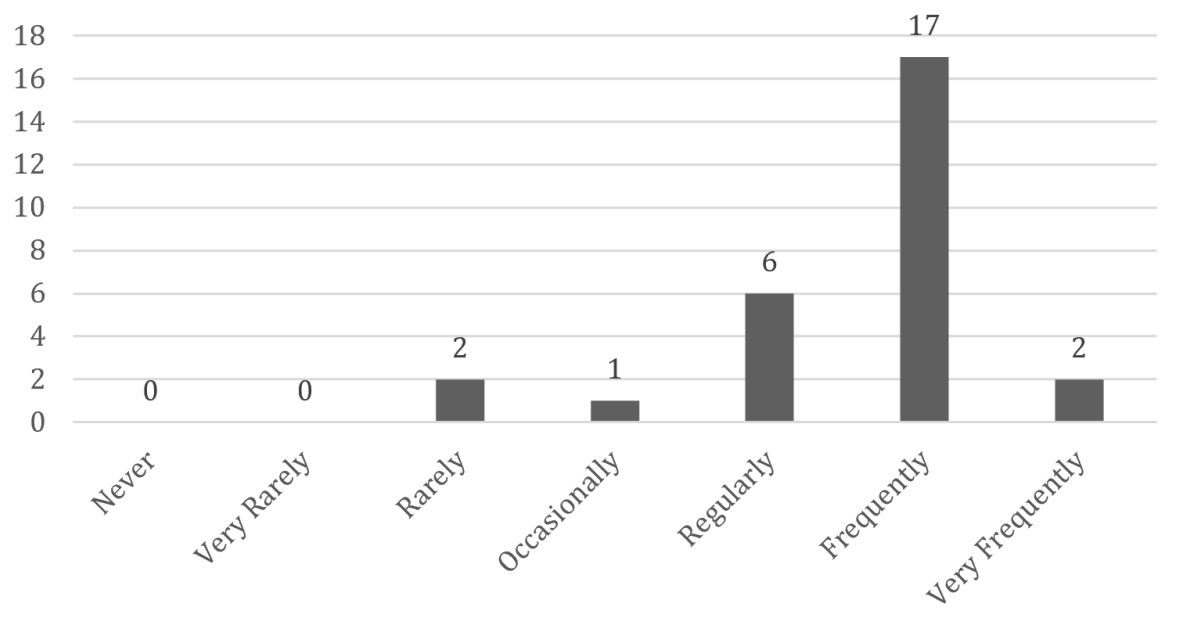

Figure 1: A frequency distribution of how often participants played games. Rarely corresponded to playing a game every few months, Occasionally to a few hours a month, Regularly to 1-2 hours a week on average, Frequently to 6 hours or more per week, and Very frequently corresponded to playing games every single day for an hour or more.

\section{Number of Games Analyzed Per Participant}

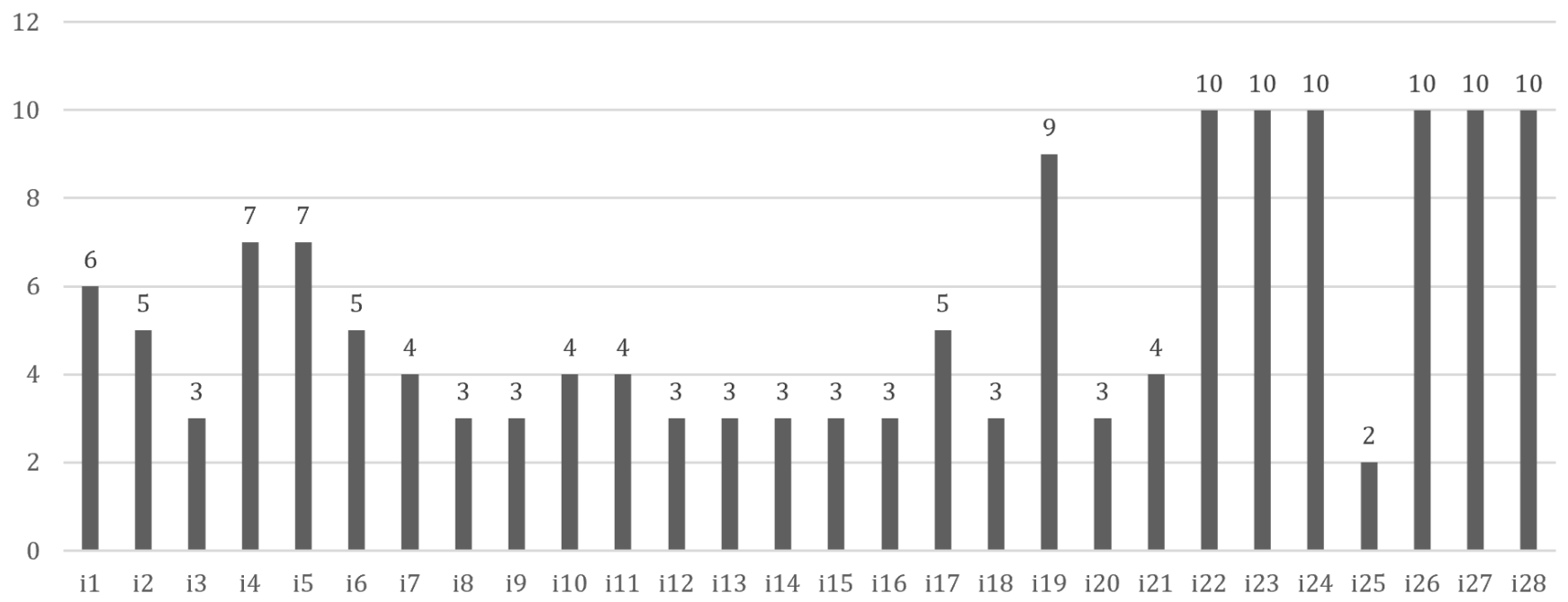

Figure 2: The number of games analyzed per participant. Participants were told that providing 3 descriptions at least was preferred, but that they could provide more descriptions if they'd like. If more than 10 games were described by the participant, on the first 10 were included in the analysis.

not use these structural terms often described the organization of the story content using concepts such as "routes", "rails", or "paths" that needed to be traversed or navigated. For example, "So, I love this game, I love it so very much, but I feel like it's a great example of not necessarily railroading, but a strict path you basically all but have to follow" (i12, Ape Escape 3).

Endings was applied when participants discussed reaching the perceived ending of the story, the perceived number of endings, and endings as a result of the player's interaction with the story over the course of one or more playthroughs. Endings was applied 45 times. For example: "I think the fact that it has like six or more different endings gives you a lot of replay value, because you can go back and change your answers to 3-5 different questions and get a completely different ending" (i20, Catherine).

Choices was applied to any instances where the participant referred to having, making, or considering a choice in relation to 


\begin{tabular}{|c|c|c|}
\hline Code Name & Definition & Count \\
\hline Structure & $\begin{array}{l}\text { A participant talks about the structure of the game's main or side stories and/or the order in } \\
\text { which events are experienced }\end{array}$ & $228(22 \%)$ \\
\hline Endings & $\begin{array}{l}\text { A participant talks about the ending(s) of the story, such as how many there are, how much } \\
\text { they are impacted by interaction, and their perception of or feelings towards the ending(s) }\end{array}$ & $45(4 \%)$ \\
\hline Comparisons & $\begin{array}{l}\text { A participant compares or juxtaposes a game or its elements to other games or elements in } \\
\text { other games }\end{array}$ & $52(5 \%)$ \\
\hline Player Narrations & $\begin{array}{l}\text { A participant discusses the extent to which a game allows or enables them to tell stories based } \\
\text { on their experience, alone or with other people, or to what extent the game allows the player } \\
\text { to narrate their own experiences }\end{array}$ & $71(7 \%)$ \\
\hline Rating Statement & A participant talks about what agency rating they are giving a game & $71(7 \%)$ \\
\hline Plot Twist & $\begin{array}{l}\text { A participant talks about plot twists or surprise events that re-contextualize how they feel } \\
\text { about the story, or how they understand it and/or their interaction }\end{array}$ & $11(1 \%)$ \\
\hline Choices & $\begin{array}{l}\text { A participant talks about the choices they have in the story, and what the consequences and } \\
\text { impact of those choices are or might be }\end{array}$ & $146(14 \%)$ \\
\hline Narrative Impact & $\begin{array}{l}\text { A participant talks about whether and how they were able to impact the story or elements in } \\
\text { the story. This code also applied in situations where a participant discussed having a lack of } \\
\text { narrative impact }\end{array}$ & $109(11 \%)$ \\
\hline Social Investment & $\begin{array}{l}\text { A participant talks about social interaction in the game/story in some manner, or expresses } \\
\text { social investment or social impact in connection to their experience, the story, or elements of } \\
\text { the story world }\end{array}$ & $15(1 \%)$ \\
\hline Genre Conventions & $\begin{array}{l}\text { A participant talks about having expectations for a game due to its genre, or when they cite } \\
\text { the game's genre as an explanation or justification for something }\end{array}$ & $20(2 \%)$ \\
\hline Story Quality & $\begin{array}{l}\text { A participant talks about the quality of the story and/or how the story quality impacted their } \\
\text { experience }\end{array}$ & $84(8 \%)$ \\
\hline Mechanical Experience & $\begin{array}{l}\text { A participant talks about how mechanics and game systems impact their experience with the } \\
\text { game and the story }\end{array}$ & $190(19 \%)$ \\
\hline Emotional Investment & $\begin{array}{l}\text { A participant talks about being immersed in the game or story world in some manner, or } \\
\text { expresses an emotional connection to elements of that world }\end{array}$ & $68(7 \%)$ \\
\hline Customization & $\begin{array}{l}\text { A participant talks about how elements of the game (such as character creation or skill trees) } \\
\text { allowed for customization of the experience }\end{array}$ & $60(6 \%)$ \\
\hline Meta-Acknowledgement & $\begin{array}{l}\text { A participant refers to the game engaging in } 4 \text { th wall breaking or to scenarios where the } \\
\text { participant acknowledges either the game or the makers of the game as being a separate } \\
\text { entity in relation to their experience (such as by saying that the designers are communicating } \\
\text { something to them through the game) }\end{array}$ & $32(3 \%)$ \\
\hline Preferences & $\begin{array}{l}\text { A participant talks about their own preferences in regards to their experience with the story or } \\
\text { gameplay, as well as how their preferences impact their experience with the story or gameplay }\end{array}$ & $19(2 \%)$ \\
\hline Expectations & $\begin{array}{l}\text { A participant talks about what is expected of them (by themselves, other players, the game, or } \\
\text { the designers) or how the game builds expectations about their interaction }\end{array}$ & $56(5 \%)$ \\
\hline
\end{tabular}

Table 1: The final set of codes, their definitions, and the number of times they were applied

the story, and any projection the participant mentioned in relation to they believed the consequences and impact of those choices to be. Choices was applied 146 times. For example: "You don't get to make any choices in the plot, it does what it does. You can choose to follow subplots but you're all going to arrive at the same place eventually" (i11, Tales of Zestiria).

Narrative impact is counted as structural here as it can be understood in terms of the perceived and projected consequence of choices and actions that the player takes. The scope and broad impact of such consequences is often described in terms of structure. Thus, this code was applied whenever a participant discussed how they perceived their actions to have (or not have) an impact on the game world or story progression. Narrative Impact was applied 109 times. For example: "You can make choices that change the game a little bit, in terms of who you develop relationships with and who you want to run your farm but in the end has the same conclusion for everyone" (i2, Stardew Valley).

\subsection{Player Experience}

The "Player Experience" category encompassed four factors: Emotional Investment, Social Investment, Preference, and Rating Statement. See table 1 for code definitions. These factors all relate, to some degree, to cognitive or individual differences, and how these 
impact the experience and the participant's assessment of the narrative and their perception of agency.

Emotional investment was applied whenever a participant mentioned being impacted due to the feeling of being immersed in a game's story or world or feeling an emotional connection to some aspect of that story or world. This code also applied whenever a participant mentioned feeling as though their agency was impacted by a lack of emotional investment. This code was applied 68 times. For example: "The reason I rate it [low agency rating] as also not mattering is because the game very quickly sets you up for what you as a player want to do, with a nice loving family and the horrors that happen to them, so you're immediately attached to the characters, and invested in playing their story through"(i8, Mother $3)$.

Social investment was applied whenever a participant discussed the ways in which they felt their agency was impact by the game's ability to facilitate some kind of social experience around play. This code was applied only 15 times. For example: "And you do get some agency there, when you're there alone it doesn't really matter, but when you're in a cast of other people, it's like "what weapon am I using?" "how do I fit into the team?" "who looks cooler?" "am I level 50 and they're level 7 and I'm protecting them?" "Are they level 999 and I'm hiding behind them?" That's what makes every hunt different and what I connect with the most. It's a case of building your own story through gameplay" (i10, Monster Hunter World).

Preferences was applied whenever players discussed how their perception of agency was influenced by their own preferences towards a game, genre, or any specific element of game design (such as mechanics, characters, or story context). This code was applied only 19 times. An example of a statement in which it was implied or stated that preferences impacted perception of agency include: "You don't make choices in that game. You just do whatever they tell you. Which is fine. I like climbing on things" (i2, Uncharted).

Rating statement was applied whenever a participant talked about what agency rating they were giving a game. This could be because they explained their rating, reiterated their rating, defended their rating, or adjusted their rating. This code was applied 71 times. For example: "So, I guess it's not a perfect agency game, but it's a high agency game, because there's a lot of choices" (i16, Until Dawn).

\subsection{Player Knowledge}

The "Player Knowledge" category encompassed three factors: Genre Conventions, Expectations, and Comparison. See table 1 for code definitions. These factors relate to elements of gameplay that players are aware of from previous play or external activity, and how they use this information to reason about and compare their experiences. Specifically, how perceived agency is impacted by the extent to which the experience is seen to match existing knowledge.

Genre Conventions was applied whenever a participant talked about having expectations or feelings towards a game due to its genre. It was also applied whenever participants cited a game's genre when evaluating its design or mechanics in relation to their perceived agency. This code was only applied 20 times. For example: "It's more TellTale style where you know that there is a script and at the end of the day with all those types of games you know that there is a large branching tree in the background with all that you could possibly do, compared to like other games where you can go off and do whatever you want like Minecraft where you can do basically everything" (i6, Detroit Become Human).

Expectations was applied to instances where a participant talked about what was expected of them, what they expected of the game, or how the game built (or violated) expectations. This code was applied 56 times. For example: "It feels like it is building towards something that matters and then it doesn't" (i26, Safe House).

Comparisons referred to instances when a participant compared or juxtaposed a game or its elements to other games. This code was applied 52 times. For example: "It kind of has aspects of Minecraft except a lot of time with that game things can change or regenerate. But with Dragonquest Builders whenever you choose to alter the environment or do something in a quest it does never revert..." (i17, Dragonquest Builders).

\subsection{Story}

The "Story" category included three factors: Plot Twist, Story Quality, and Meta-acknowledgement. See table 1 for code definitions. Unlike previous categories, the three factors in this category deal almost exclusively with the content of the story, and a participant's expectations, preferences, and judgement of that content. Although story content was not the primary focus of this work, these factors were included due to the prevalence with which participants cited the content of the story as a major contributor to their sense of agency.

Plot twist was applied whenever a participant mentioned surprising moments in the plot or endings that caused them to recontextualize how they felt about, or understood, the story. This code was only applied 11 times. For example: "You spent most of the game thinking you're the good guy but then you get to the end and realize you've been assisting the enemy the whole time" (i13, Kingdom Hearts 2).

Story quality was applied whenever a participant discussed the quality of the story or the writing within the game, especially if it was discussed in relation to how their agency was impacted by the quality. This could apply to praise or critique of the quality. Story quality was applied 84 times. For example: "It does have some dialog options and a few choices that will cause some differences so its not a $100 \%$ linear, but it has a lot of narrative framing that makes structurally insignificant choices feel significant even if you know that it is not going to have a major effect on anything you want to stop and think about what you are doing because the game makes everything feel more significant and more important than just pushing the buttons to play the game" (i28, Nier: Automata).

Meta-Acknowledgement was applied in any situation where a participant refers to the game engaging in fourth wall breaking, or any scenario where the participant personifies the game or its creators as an entity that was impacting their experience. Metaacknowledgement was applied 32 times. For example: "I think another interesting thing is that, it actually does actively strip away your agency, with the game freezing and the character calling you out as the user on your computer not just the character in the game" (i21, Doki Doki Literature Club). 


\subsection{Mechanics}

Mechanics consists of two factors: Mechanical Experience and Customization. See table 1 for code definitions. Both codes relate to how a player's perceived agency is influenced by the mechanical elements of a game's design. Customization refers specifically to moments when a participant discussed how mechanical customization options, such as character creators or personalize-able skill trees, impacted their sense of agency. Mechanical experience refers more generally to any discussion of the intersection between game mechanics, especially those not inherently tied to narrative progression, and perceived agency.

Mechanical experience was applied whenever a participant discussed how mechanics and game systems impacted their experience with the game and the story. This included a large variety of mechanical experiences relating to avatars, characters, equipment, load-outs, and gameplay, as well as freedom and variety offered by in-game systems, game mechanics, as well as in content and activities relating to them. This code was applied 135 times, making it the third most applied code. For example: "I was able to choose any character I wanted, there is a lot of freedom in it because of the various abilities and you can pick whatever you want. Powers, weapons, pets, everything was customizable" (i5, Warframe).

Customization was applied whenever a participant discussed how elements of the game allowed for customization of the experience. These statements typically referred to customize-able mechanical elements, such as skill trees or a character creator. Customization was applied 54 times. For example: "[Street Fighter 4] had a really detailed customization option, you can't do that with [Street Fighter 5] you have to buy the costumes, you can't make your own" (i21, Street Fighter 5).

\subsection{Player Narrations}

Player Narrations refers to moments when players discussed how they could go on to tell others about their gameplay experience or compare it to someone else's. See table 1 for code definition. In many cases, participants expressed the importance of their experience being unique when compared to that of others. This code is in its own category because it is referring to an evaluation process for gauging the experiential variety that the game affords. It is not about individual elements or how they feel about the narrative, but rather about how holistically the game is affording a particular set of processes or behaviors that will allow them to realize their goals.

Player Narrations was applied 31 times. For example: "There are completely non-story bits of it, where everyone has some story of something that they did that was the result of them trying to abuse the system like 'what do I do if I tie balloons to this raft' or 'if I put these magnets together can I build a hover craft'." (i10, The Legend of Zelda: Breath of the Wild).

\subsection{Concept Overlaps}

In the context of this work, a concept overlap was identified when a code disagreement was consistent across the data set. For example, if the first researcher applied "choices" to a number of excerpts, but the second researcher consistently applied "mechanical experience" instead, this would be identified as a concept overlap between the two codes. This does not refer to any one code being more correct than others, and there is no ground truth, rather, it is a sign of disagreement as a result of conceptual overlaps in the data set. For example, in the case of Choices and Mechanical Experience, participants perceived mechanical interaction, rather than just plot decisions, as a type of choice, for example "Dramatically open world design gives you mechanically a lot of choice of what to do at any moment" (i10, The Legend of Zelda: Breath of the Wild). Such differences in interpretation indicate that the coders perceive a connection between the concepts. A diagram of all concept overlaps can be seen in fig. 3 .

There were two codes that saw overlap across all 6 categories: Structure and Mechanical Experience. Both codes refer to observable design features; Game mechanics are directly observable and structure judgements are based on observable features such as choice selection. Mechanical Experience and Structure overlapped with codes that reflected more abstract and cognitive elements of the player's experience, such as expectations, player narrations, or narrative impact. These two main codes represent observable design elements that can be used to exemplify, justify, or reason about the player experience or how player knowledge impacts that experience. However, because experience and knowledge are more difficult for the player to pick apart, participants tended to fall back on the more concrete and observable, which in this case are the mechanics and the structure. In other words, participants tended to use observable design elements to reason about or justify abstract elements of their experience.

One example of this phenomenon can be seen where Player Narrations overlap with Mechanical Experience. As previously discussed, some participants judged their agency based on whether or not a game afforded them the ability to create a unique narrative experience. This is an abstract concept that connects a game's design affordances to future re-tellings of the gameplay experience. Participants often did not have the words to describe this particular connection, or how it impacted their perception of agency. Instead, participants often discussed this concept in relation to the game's mechanics. The game's mechanical systems, as a tangible representation of the game's affordances, were recognizable to players as an element that afforded them the ability to create such narrations, and therefore could be used to explain the sensation. For example: "It's an MMO RPG, with a pretty well developed world and storyline [...] But with the RPG elements there is a lot of customization and options that allow you to really make the game your own" (i4, Warframe). This overlap also manifested in the case of Customization, in which mechanics allowed for customization, which in turn facilitated player narrations. For example: "The upgrade skill tree path was where I felt the most agency. Getting to pick which tree to pick whether I wanted to be a stealth baddass or strong baddass" (i3, Bioshock).

\section{LIMITATIONS}

The study presented here is an exploratory qualitative study. The data was collected from 28 participants and covered a large range of games. There was no one game that was described by a substantial number of participants. Of 118 games, 91 games were discussed by only participant, 20 were discussed by two, 4 were discussed by three, and only two, Nier: Automata and The Witcher 3, were 


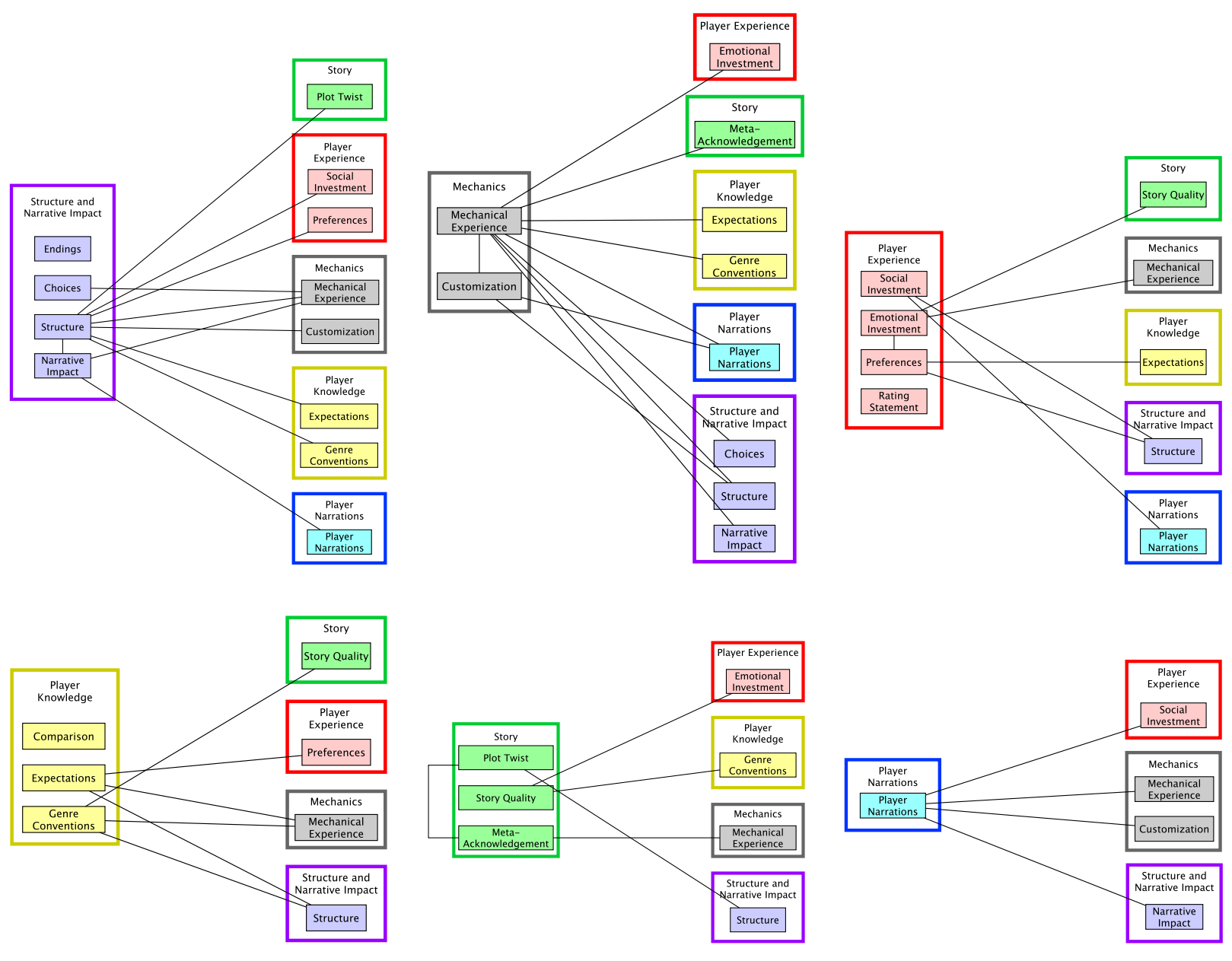

Figure 3: The 17 factors identified in the study to impact perception of player agency conceptually overlap with each other. Most common were overlaps with factors from the Structure and Mechanical experience categories. This figure shows overlaps for each category.

discussed by four participants each. As a result, it is difficult to make any conclusive claims regarding how individual differences impact perceptions of agency. However, the work presented here provides a number of factors that can be used as a stepping stone towards exploring that question in greater detail. Similarly, while the population included some international participants, it was predominantly focused on players from the United States. We acknowledge that cultural differences would likely influence agency perception, and argue that this work can act as a stepping stone to future studies that examine this phenomenon.

The interview required the participants to recall and report on their agency experience for games they had played in the past. Recalling their experience might have impacted, not only their agency rating for a given game, but also how they evaluated the games that they subsequently rated in the interview. Participants were allowed to change their ratings retro-actively, which was not documented. Thus, it is not clear to what extent this has impacted the results and whether ratings would remain consistent over a longer period of time or whether they would change depending on what games were recalled and in what sequence. Further, it is unclear whether these ratings were consistent with those players initially had during and at the moment of completing the final play session for the game. As such, future studies on player agency in games should account for memory, sequence of recall, comparison, and previous player experience and knowledge of games. We plan to examine this further in future work.

\section{DISCUSSION}

\subsection{Agency Beyond Structure}

Structural elements, i.e. choices, endings, narrative impact, and structure types, have been some of the most frequently discussed and studied design elements in relation to the study of narrative agency in the literature e.g. $[4,5,13]$. They also feature prominently in our findings. 
Participants heavily reference and mention them in relation to their perception of agency. As expected, the amount of narrative impact described by the participants often translated into the rating, i.e. having no to low perceived impact on the story was often associated with low agency rankings, and higher perceived impact on the story was more associated with higher ratings. However, when elements associated with high narrative impact like branching structures were not present, i.e. in linear games like those in the Pokemon franchise, the agency rating was not necessarily lowered. In many cases, elements of the game's mechanical design could bolster a feeling of agency even if the player could not directly control the narrative progression.

Even when the plot line was seen as completely linear and players had no perceived control over the narrative progression, many games featured mechanics that afforded players a sense of control that bolstered their feelings of agency. In fact, many attributed mechanical elements to "lifting" or improving their agency rating for games that featured linear story-lines or limited to no narrative impact. For example: "So in the game whenever you make a change to the world it stays there, it does not change, it's permanent. [...] you end up guiding how the world ends up. It still has a narrative that forces you along but there are a lot of variables that are dependent on the player. So I wouldn't say it's low, you have a good amount of power but I wouldn't say it's totally on you. I wouldn't rate it high because the story line is pretty strict" (i17, Dragonquest Builders). A key example of how this manifested was via descriptions of how mechanics and related design elements afforded different types of customization. Many participants cited the ability to customize their avatar, their in-game settings, or their combat abilities or load-outs as a key element of their sense of agency, even in linear narratives. For example: "There's a set story-line but you have customization options for our combat set up and the order that you can visit the worlds and whatnot" (i7, Kingdom Hearts: Birth by Sleep). Customization has been shown to impact player autonomy in games [19]. As autonomy is closely related to the construct of agency, our findings lend further support to customization being a factor contributing to a player's perception of control.

In addition to bolstering the sense of agency in linear narratives, mechanical experience could also create an increased sensation of agency when game mechanics were perceived to be directly linked to a player's narrative impact. Or, conversely, if mechanics were adjusted or restricted based on a narrative event. In other words, when mechanics and structure were seen as integrated and complementary to each other. For example: "It also does a good job of having the mechanics reflect narratively what is happening. The player character has a virus for example and you have to move her across the map but she can't run or jump so you have to walk her and it was really difficult. Similarly there is a part where you are fighting enemies that are shooting EMPs and if you get hit the your screen "malfunctions" and it is difficult for you to see" (i28, Nier: Automata).

The way players describe the integration between the mechanics and the narrative, implies a degree of knowledge beyond what the game itself provides. Initially its own code, during the iterative coding process we found that players were not consistent in how they described this meta-knowledge or its application. This lead to inconsistent application of the code and its eventual merger with Mechanical Experience.

In fact, the Mechanical Experience code is a merger of three codes: "Mechanical Experience", "Mechanical Meta-Knowledge", and "Narrative Mechanical Interaction". The three codes were merged during the iterative coding process due to participants' descriptions containing prominent overlap between the three concepts, making them difficult to distinguish and code consistently. Future work should examine this factor in more detail to further break down and identify elements of interest, so that participant descriptions can be coded and analyzed in a more nuanced capacity.

\subsection{Agency Evaluation Processes}

As previously discussed, our findings show that perception of agency depends on more than choices and their impact on possible endings. As a result, the different processes participants used to evaluate their agency leveraged aspects of the gameplay experience beyond structural design elements. Two of the most prominent concepts associated with these evaluation processes were Expectations and Player Narrations.

6.2.1 Expectations. Expectations are a central consideration for player agency $[32,38]$ but to the authors' knowledge have yet to be studied in relation to player perception of narrative agency. In our study, participants rarely explicitly described their expectations but instead almost always discussed or referenced expectations implicitly as associated with another element or concept captured by other codes. One example of this can be seen in the way that participants implied that the presence of choices generated an expectation for certain outcomes. For instance: "I think my biggest annoyance is giving me choices that very obviously don't matter and you are just giving me a choice to give me a choice, like that bar scene if its going to go that way no matter what. Don't give me the option and not let me do it" (i28, The Wolf Among Us).

Expectations, and the extent to which a game met or defied them, was often used as a measure to evaluate agency. This was frequently described in conjunction with a comparison to another title or franchise. Further, comparisons were often used by participants to establish baselines or points of reference through which games were ordered in terms of perceived agency. For example: "There's side quests, but you really can't ignore the main story, the focus is solidly on the main story and you can do side quests and there are some story things, but I feel like it is intended for you to have your focus on the main story, vs. like Skyrim, Horizon Zero Dawn, Zelda, where like $50 \%$ of it is on the main story and $50 \%$ of it is like, running around and doing whatever you want or fighting Ganon naked and on fire if you want or something” (i7, Nier: Automata). Participants often mention specific design elements or dynamics and directly compare them across games, and use their existence or absence as justification. For example: "Going to agency, it's who you talk to and how you want your personality to be, what you say, so that's why its medium. Comparing it to point and click having medium agency, but something like, Zelda where you can do whatever and having quest and stuff, I think of those as high agency games where you can do and go wherever you want. You can just ride a horse if you want and go anywhere, but in this game 
and in Broken Age you can only do one simple task over and over again" (i18, Softly with Teeth).

Notably, some participants would ask to change their initial rating for a game while in the midst of comparing it to another game. Such rating changes were not counted nor made a note of as a part of the protocol as they were not included in the interview data. However, this phenomenon was sufficiently prevalent to be observed and noted independently by both interviewers. This use of comparisons by participants to describe their agency experiences has interesting implications for how player agency is perceived and more importantly evaluated by players. This suggests the possibility that players do not necessarily have a fixed idea of what high or low agency entails and evaluate this in context with the game itself and their experience and knowledge of games that they consider to be similar or of the same genre.

6.2.2 Player Narrations. Player narrations also emerged as a measure by which participants evaluated agency. Specifically, participants judged a game's agency based on the possibilities it afforded them to narrate a unique experience. For example: “...the game is built of this system with all these physics and interactions, and playing with this set of rules you can create interesting, unexpected consequences, and I think everyone comes out of it with these unique experiences that they had and that's almost as much a part of the story as anything else" (i10, The Legend of Zelda: Breath of the Wild). The ability to experience something unique from other players was of particular importance, as illustrated by: "You can navigate really easily, and you have a lot of options of what puzzles you want to solve first and what you want to investigate first so I feel like everyone who plays it has a unique experience" (i21, Nine Hours, Nine Persons, Nine Doors).

Further, the ability to roleplay, and how player narrations played into that phenomenon, was used to evaluate agency. Here, participants expressed a need for being able to adjust the mechanics and story such that they could achieve a particular narrative goal. For example: "The narrative is mostly told through small amount of dialog and procedurally generated text, but you can create your own internal monologue of your own narrative and many people do when playing the game and the freedom that they find to work within the sketchy outlines of the narrative and build something more complete and full-fledged for their character and make up a narrative that makes sense to them, is for me a high degree of agency, because you can control what your character is and who they are, what they are trying to do, what their physical form etc" (i27, Caves of Qud).

This indicates that agency is evaluated not only based on what happens during gameplay, but also in how the game affords variation of experience that will enhance the act of narrating that experience to others. In addition, this serves as a measure of comparison, where players can discuss and compare their experiences. A wider range of variation in the narrations indicates the variety of interactions within the game, which in turn indicates the game's perceived agency.

\subsection{Individual Differences}

The descriptions show that player perception of agency is subject to individual differences. The most illustrative examples of this are where player descriptions of the same game rate and describe the game in very different ways. These examples fell into two categories: perceiving different design elements of the same game as impacting their perceived agency in different ways, and perceiving the same design elements of the same game as impacting their perceived agency in different ways.

In the case of the first category, participants would sometimes give the same game different ratings, and cite different elements of the game's design as the main contributors to their perceived agency. An illustrative example of this can be seen with the game Pokémon Emerald. Participant i6 rated the game high, stating "To be honest I had no agency in the story. To be fair that is the game, you kind of walk through it. In terms of the game you have more agency but in terms of the story there is actually none. The mechanics feel like they have a lot of agency. There are all these different teams and they can have all these different moves and stuff, but in terms of the story, its just... go through it. The agency is not in the story it's in the mechanics of the game." By contrast, participant i8 rated the game low: "You can actually feel impacted by that low agency. As expected from a Pokémon game, you really don't have a lot of narrative choices, that's just how it is. The reason I would say that the lack of agency is a bigger problem here, is because the game presents you with many things that your character should've been able to do, but because of the narrative design, you can't. When you play the game, the only thing you can do is become a Pokémon master and fight the bad guys and become the champion." Here both agreed on the low narrative impact players had in the story, however i6 emphasized the agency they perceived to gain from the mechanical elements of the game, leading to a high rating. Participant i8 however, emphasized the low narrative impact over other elements, disregarding the mechanics, leading to a low rating.

In the case of the second category, participants seem to agree on which design elements and structural components impact the experience but disagree on how they impact perceived agency; arguing for opposite ratings for the same game. An illustrative example of this can be seen in the case of The Wolf Among Us, which received direct opposite rankings (high and low) by two separate participants. Participant i23 rated the game as having high agency: "Even though it branches off and then back in and things don't matter, it feels like it matters. You consider the impact and consequences of your actions, which is powerful. And they are shown to impact the characters in some ways." Participant i24, however, rates it low: "... my personal experience with the moment to moment decisions I made wasn't very, didn't feel very impactful, because every single time I could predict how they would bring it back and what was going to happen, and in the end it doesn't really matter and that Crane was the person who did the deed. It didn't matter how you would get there, ...". Both participants discuss the same elements in their argument, focusing on the game's foldback structure and choices. Participant i23 saw the structure as not giving them long term impact over the story but cites the choices as being impactful, rating the game as having high agency. Participant i24 describes similar structural elements and having choices, but rated the game as having low agency. They argued that the choices did not feel impactful partly because of the structure leading to the same eventual conclusion, an element which was discounted within i23's argument. 


\section{CONCLUSION}

There has been a lack of work examining agency from the perspective of how players perceive it. The question of how players identify agency and attribute it to the design of an interactive narrative experience is currently an open question within games. To contribute to answering this question, we conducted an exploratory semi-structured interview study with 28 participants. Participants were asked to rate narrative-driven video games they had played in terms of their perceived agency, and then asked to explain their rating. To the authors knowledge this is the first phenomenological study of how players broadly perceive narrative agency and the first study to broadly explore the different ways players attribute narrative agency to various design elements in games.

The results of the study, obtained through thematic analysis of the interview data and iterative coding, identify 17 distinct factors that contribute to players' perceptions of agency, as well as ways in which they overlap and impact one another. These represent design elements, cognitive processes, knowledge, and individual preferences through which players evaluate their agency, and our analysis suggests that these factors often influence one another. Thus, making it likely that no one factor is the single determinant for a player's perception of agency. Instead, our findings indicate that agency is constructed based on multiple factors and re-evaluated by the player before, during, and after gameplay. This lends support to further exploration of player experience in the study of agency in games, such as cognitive processes and mental models of narrative and mechanics, in particular, as they relate to communicative meaning and player expectation of narrative drama and play $[31,38]$.

Due to the interplay of these various factors, and the interactive nature of games, it is likely that the player will interpret and perceive agency whether it is consciously designed for or not. Further, the nuanced interplay between design elements and individual differences emphasizes the importance of the designer being cognizant of not only the design itself, but of the player and what they bring with them, and how the player's perception of their agency will evolve over the course of their experience.

\subsection{Implications for Designing Player Agency}

Participants often approached gameplay with pre-existing expectations for interaction, encompassing both availability and impact of interaction. This was notable in relation to genre conventions, where participants described expectations for a specific mode of gameplay or the narrative structure typical of that genre. Whether those expectations were met played a considerable role in their overall assessment of agency. As a result, we want to first emphasize the importance of designers remaining cognizant of their chosen genre and design influences. This is especially true if they make design choices likely to go against conventions and functionality established by other commercial games of that genre. Established design conventions should be leveraged or defied in an informed manner and approached with care as they play a significant part in impacting agency perception.

Participants repeatedly reported and described structure in relation to how they evaluated their agency and its scope, often in terms of their choices and impact of their actions on endings or narrative events. However, participants sometimes argued that, despite being an indicator of low agency, a linear structure did not automatically equate to low perceived agency. In these instances, structure was weighed against, and in relation to, other components of the experience, and was subjected to the players individual preferences and expectations. Participants described feeling agency when they were able to make the experience their own through mechanical means, for example by developing their player characters through skill-tree progression, role-play, and customization, and/or using these features to create a new and unique experience. Thus, we found that while structure is prominently known and understood as an indicator of agency, players used it in conjunction with other elements in their assessments.

In this manner, designers can employ a linear structure, but include customizable mechanics or minor cosmetic story impacts, to constrain interaction with the narrative itself without jeopardizing a player's overall sense of agency. In general, we advise designers to consider player agency as it relates to all aspects of the experience and be aware of how their target audience might prioritize and balance the different factors. Designers should aim to balance factors based on the target agency experience they want to achieve, and consider more than one approach to afford agency to the player to account for individual differences. Further, designers can use this approach to maximize agency perception within design constraints that might otherwise have afforded a low agency experience.

\subsection{Implications for Measuring Player Agency}

In addition to design, our findings have implications regarding how agency can be measured. First, participants often discussed the factors of Structure, Endings, and Narrative Impact through navigation metaphors, such as "path" or "destination". Even when a participant used established structural terminology, such as linear or branching, they were still described in terms of being navigable, indicating that many participants possess a mental model of narrative structure as something to be traversed. In other words, structure, choices, and endings, as the navigable shape, the means of progression, and the ultimate destination, respectively, are the manifestation of narrative impact within a game's design. This navigation terminology can be used by future research to develop a concrete way to measure player's perceived agency based on the words they use to describe their experience.

Further, we found that participants in our study frequently used comparisons to update and reflect on both individual elements and games as a whole, in order to gauge how much or little agency they believed the game afforded them. This indicates that agency is described, in some capacity, as a snapshot or evaluation under specific circumstances and might be subject to change if given new examples or prompted to reflect on previous experience. Given this phenomenon, if agency is to be studied, designers and researchers should account for this process during their study design so that confounding impact of comparison can be mitigated, should that effect not be desired. Exploring this process and how agency descriptions and perceptions evolve are a valuable next step in understanding player agency in games. 


\section{ACKNOWLEDGMENTS}

Funding for this research was provided by the National Science Foundation Cyber-Human Systems under Grant No. 1526275. The authors would like to thank Joseph Wilson for his contribution to the coding effort.

\section{REFERENCES}

[1] Mary Ann Buckles. 1986. Interactive Fiction: The Computer Storygame "Adventure". Ph.D. Dissertation. University of California, San Diego.

[2] Gordon Calleja. 2011. In-game: From immersion to incorporation. MIT Press.

[3] Rogelio E Cardona-Rivera. 2011. Narrative Affordance: Towards a model of the foreseeability and perceivability of story elements in an Interactive Narrative. (2011), 250-252.

[4] Rogelio E Cardona-Rivera, Justus Robertson, Stephen G Ware, Brent Harrison, David L Roberts, and R Michael Young. 2014. Foreseeing meaningful choices. In Tenth Artificial Intelligence and Interactive Digital Entertainment Conference.

[5] Elin Carstensdottir, Erica Kleinman, and Magy Seif El-Nasr. 2019. Player interaction in narrative games: structure and narrative progression mechanics. In Proceedings of the 14th International Conference on the Foundations of Digital Games. 1-9.

[6] Kathy Charmaz. 2006. Constructing grounded theory: A practical guide through qualitative analysis. Sage.

[7] Paul Cheng. 2007. Waiting for Something to Happen: Narratives, Interactivity and Agency and the Video Game Cut-scene.. In DiGRA Conference.

[8] Doug Church. 2006. Formal abstract design tools. The Game Design Reader: A Rules of Play Anthology. MIT Press, Cambridge (2006).

[9] Jacob Cohen. 1960. A coefficient of agreement for nominal scales. Educational and psychological measurement 20, 1 (1960), 37-46.

[10] Tom Cole and Marco Gillies. 2019. Thinking and Doing: Challenge, Agency, and the Eudaimonic Experience in Video Games. Games and Culture (2019) https://doi.org/10.1177/1555412019881536

[11] Timothy Day and Jichen Zhu. 2017. Agency informing techniques: Communicating player agency in interactive narratives. In Proceedings of the 12th International Conference on the Foundations of Digital Games. 1-4.

[12] Matthew William Fendt, Brent Harrison, Stephen G Ware, Rogelio E CardonaRivera, and David L Roberts. 2012. Achieving the illusion of agency. In International Conference on Interactive Digital Storytelling. Springer, 114-125.

[13] Matthew William Fendt, Brent Harrison, Stephen G. Ware, Rogelio E. CardonaRivera, and David L. Roberts. 2012. Achieving the Illusion of Agency. In Interactive Storytelling (Lecture Notes in Computer Science). Springer, Berlin, Heidelberg, 114125. https://doi.org/10.1007/978-3-642-34851-8_11

[14] Francesca Foffano and David Thue. 2019. Changes of user experience in an adaptive game: a study of an AI manager. In Proceedings of the 14th International Conference on the Foundations of Digital Games. 1-8.

[15] Helen Gavin. 2008. Thematic analysis. Understanding research methods and statistics in psychology (2008), 273-282.

[16] Saumya Gupta and Theresa Jean Tanenbaum. 2019. Evaluating the Pleasures of Agency in Shiva's Rangoli, a Tangible Storytelling Installation. In Proceedings of the 2019 on Designing Interactive Systems Conference. 49-60.

[17] Sean Hammond, Helen Pain, and Tim J Smith. 2007. Player agency in interactive narrative: Audience, actor \& author. (2007).

[18] D Fox Harrell and Jichen Zhu. 2009. Agency Play: Dimensions of Agency for Interactive Narrative Design.. In AAAI spring symposium: Intelligent narrative technologies II. 44-52.
[19] Keunyeong Kim, Michael G Schmierbach, Mun-Young Chung, Julia Daisy Fraustino, Frank Dardis, Lee Ahern, et al. 2015. Is it a sense of autonomy, control, or attachment? Exploring the effects of in-game customization on game enjoyment. Computers in Human Behavior 48 (2015), 695-705. https: //doi.org/10.1016/j.chb.2015.02.011

[20] J Richard Landis and Gary G Koch. 1977. The measurement of observer agreement for categorical data. biometrics (1977), 159-174.

[21] Brian Magerko and John E Laird. 2003. Building an interactive drama architecture. In First International Conference on Technologies for Interactive Digital Storytelling and Entertainment. 226-237.

[22] Bride Mallon. 2008. Towards a taxonomy of perceived agency in narrative gameplay. Computers in Entertainment (CIE) 5, 4 (2008), 1-15.

[23] Stacey Mason. 2013. On games and links: Extending the vocabulary of agency and immersion in interactive narratives. In International Conference on Interactive Digital Storytelling. Springer, 25-34.

[24] Michael Mateas. 2001. A preliminary poetics for interactive drama and games, In Digital Creativity. Digital Creativity 12, 3, 140-152.

[25] Alex Mitchell, Liting Kway, Tiffany Neo, and Yuin Theng Sim. 2020. A Preliminary Categorization of Techniques for Creating Poetic Gameplay. Game Studies 20, 2 (2020).

[26] Janet Horowitz Murray. 1997. Hamlet on the Holodeck: The Future of Narrative in Cyberspace. The Free Press, New York, NY, USA.

[27] Ashwathy T Revi, David E Millard, and Stuart E Middleton. 2020. A Systematic Analysis of User Experience Dimensions for Interactive Digital Narratives. (2020), 58-74.

[28] Mark Riedl, Cesare J Saretto, and R Michael Young. 2003. Managing interaction between users and agents in a multi-agent storytelling environment. In Proceedings of the second international joint conference on Autonomous agents and multiagent systems. 741-748.

[29] David L Roberts, Merrick L Furst, Brian Dorn, and Charles L Isbell. 2009. Using influence and persuasion to shape player experiences. In Proceedings of the 2009 ACM SIGGRAPH Symposium on Video Games. 23-30.

[30] Sarah Stang. 2019. This Action Will Have Consequences': Interactivity and Player Agency. Game Studies 19, 1 (2019).

[31] Karen Tanenbaum and Theresa J Tanenbaum. 2009. Commitment to meaning: a reframing of agency in games. In Digital Arts and Culture Conference (DAC 2009).

[32] Karen Tanenbaum and Theresa Jean Tanenbaum. 2010. Agency as commitment to meaning: communicative competence in games. Digital Creativity 21, 1 (March 2010), 11-17. https://doi.org/10.1080/14626261003654509

[33] Suzanne C Thompson, Wade Armstrong, and Craig Thomas. 1998. Illusions of control, underestimations, and accuracy: a control heuristic explanation. Psychological bulletin 123, 2 (1998), 143.

[34] David Thue, Vadim Bulitko, Marcia Spetch, and Trevon Romanuik. 2010. Player agency and the relevance of decisions. In foint International Conference on Interactive Digital Storytelling. Springer, 210-215.

[35] David Thue, Vadim Bulitko, Marcia Spetch, and Trevon Romanuik. 2011. A computational model of perceived agency in video games. In Proceedings of the Seventh AAAI Conference on Artificial Intelligence and Interactive Digital Entertainment. 91-96.

[36] Matti Vuorre and Janet Metcalfe. 2016. The relation between the sense of agency and the experience of flow. Consciousness and cognition 43 (2016), 133-142.

[37] Noah Wardrip-Fruin. 2020. How Pac-Man Eats. MIT Press.

[38] Noah Wardrip-Fruin, Michael Mateas, Steven Dow, and Serdar Sali. 2009. Agency Reconsidered. In Proceedings of DiGRA 2009.

[39] Jichen Zhu and D Fox Harrell. 2009. Memory, Reverie Machine: towards a dance of agency in interactive storytelling. In Proceedings of the International Symposium on Electronic Art (ISEA) Conference. 